\title{
Vitamin D Levels Are Associated with Expression of SLE, but Not Flare Frequency
}

\author{
Marline L. Squance, ${ }^{1,2,3,4,5}$ Glenn E. M. Reeves, ${ }^{1,3,4,5}$ and Huy A. Tran ${ }^{1,4}$ \\ ${ }^{1}$ Faculty of Health and Medicine, University of Newcastle, Callaghan, NSW 2308, Australia \\ ${ }^{2}$ Faculty of Science and Information Technology, University of Newcastle, Callaghan, NSW 2308, Australia \\ ${ }^{3}$ Autoimmune Resource and Research Centre, 2nd Floor HAPS Building, John Hunter Hospital, New Lambton, \\ NSW 2305, Australia \\ ${ }^{4}$ Pathology North, Hunter New England, New Lambton, NSW 2305, Australia \\ ${ }^{5}$ Hunter Medical Research Institute, New Lambton, NSW 2305, Australia \\ Correspondence should be addressed to Marline L. Squance; marline.squance@hnehealth.nsw.gov.au
}

Received 30 July 2014; Revised 30 October 2014; Accepted 30 October 2014; Published 24 November 2014

Academic Editor: Ruben Burgos-Vargas

Copyright ( 2014 Marline L. Squance et al. This is an open access article distributed under the Creative Commons Attribution License, which permits unrestricted use, distribution, and reproduction in any medium, provided the original work is properly cited.

This study explores links between vitamin D deficiency $(25(\mathrm{OH}) \mathrm{D}=50 \mathrm{nmol} / \mathrm{L})$ and serological autoimmunity $($ ANA $>1: 80)$ and frequency of self-reported flares (SRF) in participants with clinical autoimmunity (SLE). 25(OH)D levels of 121 females were quantified and compared. The cohort consisted of 80 ACR defined SLE patients and 41 age and sex matched controls. Association analysis of $\log 2(25(\mathrm{OH}) \mathrm{D})$ levels and ANA 80 positivity was undertaken via two-sample $t$-tests and regression models. Significant differences were found for 25(OH)D levels (mean: control $74 \mathrm{nmol} / \mathrm{L}(29.5 \mathrm{ng} / \mathrm{ml})$; SLE $58 \mathrm{nmol} / \mathrm{L}(23.1 \mathrm{ng} / \mathrm{ml}), P=0.04), 25(\mathrm{OH}) \mathrm{D}$ deficiency $(P=0.02)$. Regression models indicate that, for a twofold rise in $25(\mathrm{OH}) \mathrm{D}$ level, the odds ratio (OR) for ANA-positivity drops to $36 \%$ of the baseline OR. No link was found between SRF-days and 25(OH)D levels. Our results support links between vitamin D deficiency and expression of serological autoimmunity and clinical autoimmunity (SLE). However, no demonstrable association between $25(\mathrm{OH}) \mathrm{D}$ and SRF was confirmed, suggesting independent influences of other flare-inducing factors. Results indicate that SLE patients have high risk of $25(\mathrm{OH}) \mathrm{D}$ deficiency and therefore supplementation with regular monitoring should be considered as part of patient management.

\section{Background}

Systemic lupus erythematosus (SLE) is a systemic autoimmune illness with a complex and multifactorial pathogenesis [1]. Patients can exhibit a wide range of symptoms including increased photosensitivity to ultraviolet radiation (UV) exposure, combined with immunological markers of antinuclear antibody positivity. It is thought that UV exposure may be a catalyst to symptom exacerbation or flare events [2-4] and therefore SLE patients are often advised to adopt sunprotective measures of using both physical and chemical barriers on a routine basis.

Although the use of UV protective measures is important as a management strategy, it is undertaken with reservation, as adopting such measures may result in vitamin D deficiency and insufficiency reducing individual patient capacity to maintain vitamin $\mathrm{D}$ synthesis within the skin. Mechanisms of UV-related influences in SLE range from induction of anti-double stranded DNA (anti-dsDNA) autoantibody production resulting in skin lesion exacerbation [3] through the possibility that vitamin $\mathrm{D}$ effects may be inhibited by SLE patient serum autoantibodies (anti-vitamin D) [5]. It is clear that vitamin $\mathrm{D}$ is an important hormone with immunomodulating properties [6-8] and has a vital role in a large number of biologic and biochemical pathways $[3,9,10]$.

Deficiency of vitamin D is also demonstrated in association with a range of immune disturbances, including allergy and autoimmune illnesses such as SLE, ulcerative colitis, rheumatoid arthritis, and multiple sclerosis [9, 11-15]. This study further explores possible links between vitamin D deficiency and autoimmunity as expressed by two parametersantinuclear antibody positivity (ANA, across normal controls 


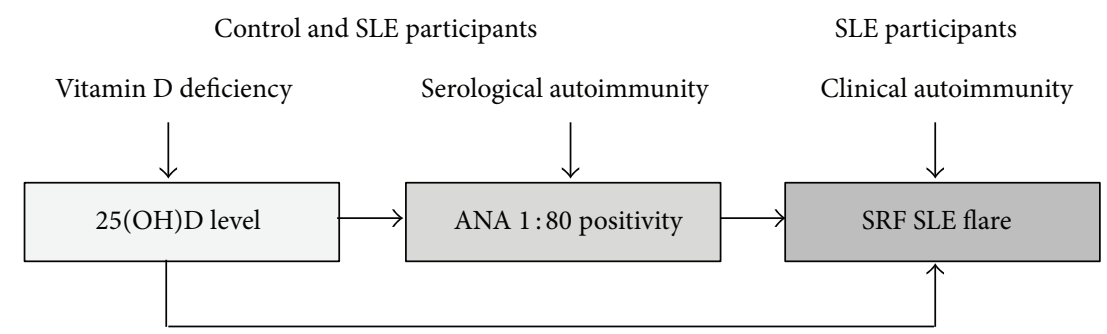

Hypothesis: vitamin D deficiency increases SRF in SLE

FIGURE 1: Proposed pathogenic pathway.

and SLE patients) and frequency of self-reported flares (SRF) in participants with confirmed autoimmune illness (SLE). Our study incorporated a group of matched controls, so that important factors modulating the risk of autoimmunity could be assessed. The proposed study's pathogenic pathway is presented within Figure 1.

In our cohort of patients with SLE, we set out to explore the determinants of flare events from a patient's perspective with the primary aim being to determine whether lower vitamin D levels were predictive of an increased risk of SRF in SLE patients. The secondary goal was to assess whether any such link was mediated by the serological expression of autoimmunity, as reflected by ANA positivity with a cutoff of ANA $1: 80$.

\section{Patients and Methods}

The overall hypothesis of this study was that, through immunomodulating activity, vitamin D (25(OH)D) levels could influence the risk of an SLE patient experiencing and reporting a flare event. The hypothesis proposes a 2step pathway: (i) from low vitamin $\mathrm{D}$ levels to expression of serological autoimmunity (expressed by ANA-positive ratio $\geq 1: 80$ ) and (ii) from ANA positivity to frequent flare events. The overall pathogenic pathway (Figure 1) examined associations between levels of 25(OH)D and SRF-days.

Vitamin D (25(OH)D) levels of a cohort of 121 female participants were quantified and compared with reference to vitamin D deficiency, insufficiency, or normal levels [3] across the cohort. Methods specific to 25(OH)D quantification and analysis are outlined below. The cohort consisted of 80 SLE patients as defined by the American College of Rheumatology (ACR) classification criteria for SLE [16] and 41 age and sex matched control study participants. Participants were Australian and of Caucasian ethnicity: they completed study specific questionnaires in regard to relevant demographics and daily living practices including hours spent in the outdoors environment and use of sun protective products of sunscreen (SS) and foundation makeup (FM). Vitamin $\mathrm{D}$ supplementation for all participants and use of other immunotherapy medications (ITM) which have photosensitizing properties such as methotrexate, hydroxychloroquine, prednisolone, and azathioprine were also recorded for SLE participants. ITM was considered as a combined group and also as individual interactive covariates. Supplementation of vitamin $\mathrm{D}$ was also considered as a separate covariate due to its reported immunomodulation properties [6, 17].

All participants had blood collected for assessment of general measures of health and immunological markers relevant to autoimmune illness and specifically SLE diagnosis, including ANA, DNA, and ENA testing. ANA positivity was recorded with ratios $\geq 1$ : 80 and used to investigate any link between vitamin $\mathrm{D}$ levels and serological autoimmunity.

SLE participants also shared their flare experience in an interview designed to examine lupus flare history over the 12-month period prior to interview. Flare data was of a self-reported retrospective nature with SRF specific methods detailed previously [18] and briefly summarised for this paper. SRF data, in particular, calculated flare days and SRF status was analysed to explore any associations with ANA positivity. Finally, possible links between vitamin D levels and likelihood of SLE flares were explored.

Ethical review and approval of study according to Declaration of Helsinki 2008 [19] were received from the University of Newcastle and Hunter New England Health Human Research Ethics Committees.

2.1. Study Population. Participants were recruited from the Hunter and Central Coast areas of New South Wales (NSW), Australia. SLE participants were recruited through the Autoimmune Resource and Research Centre (ARRC) and private immunology clinics. Inclusion criteria were specified as being 18-80 years of age and having a confirmed diagnosis of SLE. Confirmation of participant SLE diagnosis was obtained through auditing of health records according to ACR SLE classification [16]. Limited numbers of males and participants from ethnic diverse backgrounds responded to recruitment invitations, so the final study cohort reflects a female largely homogeneous Caucasian population. Control participants were recruited through the volunteer research participant database of the Hunter Medical Research Institute (HMRI). Recruitment inclusion criteria for control participants were specified as being 18-80 years of age, female, and not having an autoimmune illness.

2.2. Data Collection. As part of study specific questionnaires of a wider study (environmental determinants of lupus flares (EDOLF)), participants self-reported their personal medical history including medication and supplement use, home environment characteristics, and lifestyle practices. Of particular interest were usage patterns of ultraviolet (UV) 
protective products such as SS and FM, as well as hours spent undertaking activities in the outdoors during daylight hours.

Participants attended a scheduled study clinic appointment in the 2006 spring season (September-November) to collect questionnaires and complete study requirements including diagnostic pathology testing and assessment of relevant health measurements. Biological samples were collected by a qualified phlebotomist, processed immediately, and stored at $-20^{\circ} \mathrm{C}$ until specific analysis was required. Height and weight measures were used for calculation of body mass index [20].

SLE participants were also requested to participate in an extended structured interview to explore their personal experience and perception of their flare events. Flares were not assessed within a full physician directed clinical assessment; therefore more traditional SLE activity assessment tools (e.g., SLEDAI) could not be used. Study flare assessment involved a medical researcher following a study script inclusive of a novel flare definition [21] and a series of questions prompting recount of the number and duration of SRF, symptoms, and management over the previous 12 months. Comprehensive methods for flare assessment used in this study have been detailed elsewhere [18]. A calculation of flare days was made for the study period by multiplying the SRF number and average event length.

2.3. Foundation Makeup (FM) and Sunscreen (SS) Use. Due to the UV protective properties of FM and SS [22] and the potential relationship between use of these products and vitamin D status [23], this study collected specific data regarding participant use of these products. Product use was calculated by combining self-reported use count values within a scale of "daily (365)," "weekly (52)," "monthly (12)," "yearly (1)," and "do not know (0)." Use was reported within a home cleaning and maintenance product list (HCMPL) of the wider EDOLF study and documenting home cleaning and personal care products used over the study year. HCMPL reported products were categorised based on intended purpose of named product, with a combination category of "makeup" (FMSS) including the topical lotions of FM and SS only.

2.4. Outdoor Hours. Study year outdoor hours were calculated from participant reported estimates of average number of hours spent on a regular weekday and also weekend day in the outdoors environment.

2.5. Vitamin D (25(OH)D) Analysis. Blood samples were collected and centrifuged at $3500 \mathrm{rpm}$ for 15 minutes prior to freezing at $-20^{\circ} \mathrm{C}$. Serum was thawed prior to analysis for quantification of 25-hydroxyvitamin D $(25(\mathrm{OH}) \mathrm{D})$ via a manual radioimmunoassay (Immunodiagnostics Systems Limited, UK) using ${ }^{125}$ iodine-labelled 25-hydroxyvitamin D. The method measures total $\left(\mathrm{D}_{2}\right.$ plus $\left.\mathrm{D}_{3}\right)$ 25-hydroxyvitamin D. Serum calcium was quantified with the RXL Dimension or Vista 1500 platforms (Siemens Healthcare Diagnostics, USA). Analysis was performed in a tertiary referral laboratory, fully accredited to the standard required by the National Australian Testing Authority (NATA) (Pathology North, Hunter New England). Vitamin D status in analysis was categorised as deficiency $(50 \mathrm{nmol} / \mathrm{L}(\leq 20 \mathrm{ng} / \mathrm{mL}))$, insufficiency $(52.5-$ $72.5 \mathrm{nmol} / \mathrm{L}(>20.0 \leq 29 \mathrm{ng} / \mathrm{mL}))$, and normal $(72.5 \mathrm{nmol} / \mathrm{L}$ $(>30 \mathrm{ng} / \mathrm{mL}))[3]$.

All samples were collected within spring season of 2006 (September-November), average daily temperature $24.4^{\circ} \mathrm{C}$ (range $21.5-28.5^{\circ} \mathrm{C}$ ), and average daily global solar exposure $20.0 \mathrm{MJ} / \mathrm{m}^{2}$ (range $17.2-23.2 \mathrm{MJ} / \mathrm{m}^{2}$ ) [24]. Longitude and latitude of study area ranged between $151.16 \mathrm{E}$ and $152.01 \mathrm{E}$ and $32.68 \mathrm{~S}$ and $33.5 \mathrm{~S}$.

2.6. ANA Analysis. Antinuclear antibody testing used indirect immunofluorescence on Hep-2 slides (Immuno Concepts, Sacramento, USA) according to manufacturer's instructions, with a screening dilution of $1: 40$ and doubling serial dilution to 1:2560. Enzyme-linked immunosorbent assays (ELISA) were used to measure antibodies to DNA (Trinity Biotech, Co. Wicklow, Ireland) and ENA (Euroimmun, Lübeck, Germany), with assays performed on the automated Trituras platform (Diagnostic Grifols, Barcelona, Spain).

2.7. Other Risk Factors. Information on participant demographics, comorbid medical history, and general health and wellbeing were collected along with SLE participant nominated date of diagnosis which was crosschecked within the health record audit. Participant socioeconomic status (SES) as optioned categories of "above Australian average," "Australian average," and "below Australian average" was also collected. Current smoking status was captured as a dichotomised "yes" or "no" response and crosschecked with serum cotinine testing.

2.8. Statistical Methods. Demographic information of participant groups was summarised with the use of simple descriptive statistics. Associations between participant groups and independent variables were analysed via one-way ANOVA for continuous variables and Fisher's exact test of independence for dichotomous variables. Separate analysis was performed for use of vitamin $\mathrm{D}$ supplementation and $25(\mathrm{OH}) \mathrm{D}$ level statuses. Shapiro-Wilk testing did not confirm Gaussian distribution; therefore, to allow intuitive interpretation of any association, data was normalised through log2 transformation of $(25(\mathrm{OH}) \mathrm{D})$ levels. Association analysis of log levels and ANA 80 positivity was undertaken via two-sample $t$-test with unequal variances, as well as linear regression with dependent variable $\log 2(25(\mathrm{OH}) \mathrm{D})$ levels and logistic regression with ANA positivity as a binary dependent variable.

SLE participant SRF-days and 25(OH)D level relationship was assessed with negative binomial regression models due to overdispersion of flare data. All variables of interest were included in the initial model with a backward stepwise approach with covariates of age; diagnosis years; ACR criteria, outdoors hours; SES; BMI; FMSS, vitamin D supplementation, use of hormones, and ITM. All significant $P$ values $(\leq 0.05)$ were noted and retained in models with associations expressed as incidence rate ratios (IRR) with $95 \%$ confidence intervals. All analysis was performed using STATA v11.0 [StataCorp LP, College Station, Texas, USA]. 
TABLE 1: Characteristics of participant groups.

\begin{tabular}{|c|c|c|c|}
\hline & $\begin{array}{l}\text { Control } \\
(N=41)\end{array}$ & $\begin{array}{c}\text { SLE } \\
\text { ACR 4+ } \\
(N=80)\end{array}$ & $\begin{array}{c}\text { Difference } \\
\text { between } \\
\text { groups }\end{array}$ \\
\hline & Mean \pm SD & Mean \pm SD & $P$ value \\
\hline Age (years) & $49.8 \pm 12.4$ & $47.7 \pm 13.5$ & 1.0 \\
\hline Diagnosis (years) & & $7.7 \pm 6.2$ & \\
\hline Outdoor hours per year & $718.2 \pm 506$ & $490.5 \pm 433$ & 0.99 \\
\hline Body mass index score & $25.9 \pm 4.8$ & $27.4 \pm 5.6$ & 1.0 \\
\hline $\begin{array}{l}\text { Makeup (FMSS) days } \\
\text { per year }\end{array}$ & $307 \pm 227$ & $291 \pm 214$ & 0.99 \\
\hline $\begin{array}{l}\text { Vitamin D 25(OH)D } \\
\mathrm{ng} / \mathrm{mL}\end{array}$ & $29.5 \pm 8.2$ & $23.1 \pm 6.3$ & 0.04 \\
\hline \multirow[t]{2}{*}{$\begin{array}{l}\text { Vitamin D 25(OH)D } \\
\mathrm{nmol} / \mathrm{L}\end{array}$} & $73.7 \pm 20.6$ & $57.8 \pm 15.7$ & 0.04 \\
\hline & no. (\%) & no. (\%) & $P$ value \\
\hline Educational background & & & 0.43 \\
\hline Socioeconomic status & & & 0.48 \\
\hline Above average & $10(24.4)$ & $15(18.8)$ & \\
\hline Average & $29(70.7)$ & $56(70.0)$ & \\
\hline Below average & $2(4.9)$ & $9(11.3)$ & \\
\hline Current smoker & $1(2.4)$ & $6(7.5)$ & 0.42 \\
\hline Regular sun & $28(68.3)$ & $39(48.8)$ & 0.05 \\
\hline Use sunscreen & $32(78)$ & $65(81.3)$ & 0.81 \\
\hline $\begin{array}{l}\text { Use immunotherapy } \\
\text { medications }\end{array}$ & & $67(83.8)$ & \\
\hline $\begin{array}{l}\text { Use of vitamin D } \\
\text { supplements }\end{array}$ & $11(26.8)$ & $\begin{array}{c}29(36.3) \\
0\end{array}$ & 0.32 \\
\hline $\begin{array}{l}\text { Use of hormone } \\
\text { supplements }\end{array}$ & $10(24.4)$ & $30(37.5)$ & 0.16 \\
\hline Deficient 25(OH)D & $6(14.6)$ & $28(35.0)$ & 0.02 \\
\hline Insufficient 25(OH)D & $19(46.3)$ & $39(48.8)$ & 0.85 \\
\hline Abnormal 25(OH)D & $25(61)$ & $67(83.8)$ & 0.01 \\
\hline $\begin{array}{l}\text { Deficient } 25(\mathrm{OH}) \mathrm{D} \\
\text { with supplements }\end{array}$ & $2(4.9)$ & $13(16.3)$ & 0.09 \\
\hline $\begin{array}{l}\text { Insufficient 25(OH)D } \\
\text { with supplements }\end{array}$ & $1(2.4)$ & $15(18.8)$ & 0.01 \\
\hline $\begin{array}{l}\text { Abnormal 25(OH)D } \\
\text { with supplements }\end{array}$ & $3(7.3)$ & $23(28.8)$ & 0.01 \\
\hline$A N A \geq 1: 80$ ratio & $17(41.5)$ & $63(78.8)$ & 0.00 \\
\hline
\end{tabular}

Vitamin D categories: deficiency $(50 \mathrm{nmol} / \mathrm{L}(\leq 20 \mathrm{ng} / \mathrm{mL}))$, insufficiency $(52.5-72.5 \mathrm{nmol} / \mathrm{L}(>20.0 \leq 29 \mathrm{ng} / \mathrm{mL}))$, and abnormal $(\leq 72.5 \mathrm{nmol} / \mathrm{L}$ $(\leq 29 \mathrm{ng} / \mathrm{mL}))[3]$.

\section{Results}

Demographic data for the 80 SLE participants and 41 controls (without autoimmune illness) are shown in Table 1 along with analysis of difference between groups (with $P$ values). Groups were found to be well matched with no significant differences found in demographic characteristics. Participant mean age was 50 years for control group and 48 years with mean disease duration of 7.7 years (SD 6.2) for the SLE group. The majority of participants were Caucasian (97.5\%) with 2
SLE participants and a single control participant identifying with Asian ethnicity. Both groups within the cohort reported educational levels to advanced or vocational level and above (62\%), and an SES of either "above Australian average" or "Australian average" was reported in 110 (91\%) of the combined cohort. Participants identifying as a current smoker did differ in percentage (control 2.4\%: SLE 7.5\%) however not significantly with Fisher's exact analysis. UV exposure through sunlight has impacts on vitamin D levels; therefore participants were asked whether they spent regular periods in the sun and also asked to estimate the average weekday and weekend hours spent in the outdoors (combined into an estimate of annual outdoor hours). Within our cohort, $68 \%$ of control participants as compared to $49 \%$ SLE participants reported that they experienced regular periods in the sun. This resulted in a calculated significant difference between the groups $(P=0.05)$. However this difference was not found when comparing the groups' calculated estimate of annual outdoor hours. The use of SS was reported in our cohort in similar proportions (control 78\%:SLE 81\%) reflecting general adherence to sun protective measures. Individual SS product sun protection factor (SPF) was not collected due to retrospective nature of study and multiple SS products used across the year; however health promotion advice within Australia recommends SS of a minimum SPF 30+ and the majority of SS products within Australia adhere to a minimum of SPF $30+$ standard with broad spectrum UVA and UVB protection [25].

As highlighted in Figure 2, significant differences between groups were found for vitamin D 25(OH)D levels (mean: control $74 \mathrm{nmol} / \mathrm{L}(29.5 \mathrm{ng} / \mathrm{mL}) ;$ SLE $58 \mathrm{nmol} / \mathrm{L}$ (23.1 ng/mL), $P=0.04), 25(\mathrm{OH}) \mathrm{D}$ deficiency $(P=0.02)$, and levels categorised as abnormal $(\leq 72.5 \mathrm{nmol} / \mathrm{L})$ representing vitamin $\mathrm{D}$ levels considered to be both deficient and insufficient $(P=0.01)$. Deficiency level differences between groups were not observed when vitamin $D$ supplementation use was considered; however, this did not extend to insufficient vitamin D 25(OH)D levels with insignificant differences found in unadjusted values but significant differences $(P=0.01)$ when participant use of supplementation was factored into analysis.

SLE participant individual ACR criteria were confirmed within audit phase of the study and included within association analysis to explore potential links between criteria and levels of $25(\mathrm{OH}) \mathrm{D}$. No significant differences were found for any of the 11 ACR criteria in cross analysis with $25(\mathrm{OH}) \mathrm{D}$ levels or any subcategory of $25(\mathrm{OH}) \mathrm{D}$ levels. Due to reports of leucopoenia being a potential consequence of low vitamin D levels [3] a separate analysis via Fisher exact test was undertaken; however the association was not confirmed within our cohort.

The potential for pharmaceutical regimes to also be associated with altered 25(OH)D levels was tested with reference to quantified $25(\mathrm{OH}) \mathrm{D}$ levels and also $25(\mathrm{OH}) \mathrm{D}$ categories of deficient, insufficient, and a combined abnormal level. Medications and supplements were analysed as single variables and also as combined groups of variables as in ITM. It is of interest that hormone supplementation $(P=0.01)$ and combined ITM group $(P=0.05)$ and prednisolone 

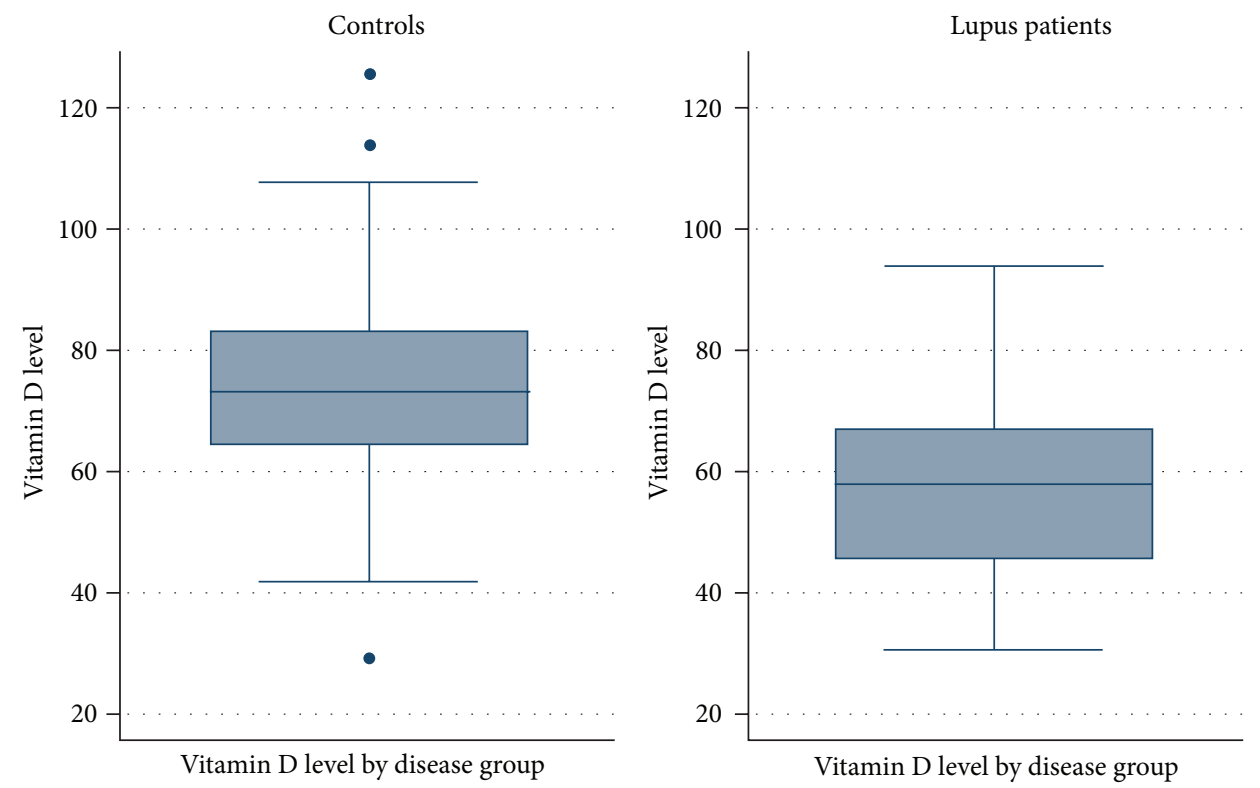

FIGURE 2: 25(OH)D level comparison controls/SLE.

TABLE 2: SLE participant differences: ACR, medications, and 25(OH)D status.

\begin{tabular}{|c|c|c|c|c|}
\hline$N=80$ & Number (\%) & $\begin{array}{c}P \text { value } \\
\text { Vit. D level }\end{array}$ & $\begin{array}{c}\text { Number ( } P \text { value }) \\
\text { Vit. D deficient }\end{array}$ & $\begin{array}{l}\text { Number }(P \text { value }) \\
\text { Vit. D insufficient }\end{array}$ \\
\hline \multicolumn{5}{|l|}{ Clinical ACR SLE features } \\
\hline Malar rash & $57(71.3)$ & 0.54 & $16(0.07)$ & $31(0.14)$ \\
\hline Discoid rash & $3(3.8)$ & 0.23 & $2(0.28)$ & $1(1.0)$ \\
\hline Photosensitivity & $43(53.8)$ & 0.52 & $11(0.07)$ & $23(0.38)$ \\
\hline Oral/nasal ulcers & $29(36.3)$ & 0.12 & $10(1.0)$ & $12(0.36)$ \\
\hline Arthritis & $63(78.8)$ & 0.63 & $21(0.58)$ & $31(1.0)$ \\
\hline Serositis & $20(25.0)$ & 0.91 & $6(0.79)$ & $10(1.0)$ \\
\hline Renal disorder & $37(46.3)$ & 0.49 & $16(0.17)$ & $15(0.19)$ \\
\hline Neurological disorder & $33(41.3)$ & 0.67 & $12(1.0)$ & $17(0.82)$ \\
\hline Haematological disorder & $39(48.8)$ & 0.95 & $14(1.0)$ & $20(0.82)$ \\
\hline Leucopoenia & $18(22.5)$ & 0.57 & $7(0.78)$ & $10(0.6)$ \\
\hline Immunologic disorder & $27(33.8)$ & 0.42 & $11(0.47)$ & $14(0.81)$ \\
\hline Antinuclear antibody $\geq 1: 80$ & $63(78.8)$ & 0.27 & $24(0.39)$ & $28(0.18)$ \\
\hline \multicolumn{5}{|l|}{ Medications } \\
\hline Hormones & $30(37.5)$ & 0.23 & $5(0.01)$ & $16(0.65)$ \\
\hline ITM & $67(83.8)$ & 0.56 & $20(0.05)$ & $36(0.07)$ \\
\hline Prednisolone & $34(42.5)$ & 0.31 & $7(0.03)$ & $21(0.07)$ \\
\hline Vitamin $\mathrm{D}+$ prednisolone & $15(18.8)$ & 0.71 & $4(0.56)$ & $9(0.4)$ \\
\hline
\end{tabular}

Vitamin D categories: deficiency $(50 \mathrm{nmol} / \mathrm{L}(\leq 20 \mathrm{ng} / \mathrm{mL}))$ and insufficiency $(52.5-72.5 \mathrm{nmol} / \mathrm{L}(>20.0 \leq 29 \mathrm{ng} / \mathrm{mL}))[3]$.

$(P=0.03)$ were all associated with deficient levels of vitamin D. Association $P$ values between 25(OH)D level, ACR criteria analysis, and medications of interest are outlined in Table 2.

Vitamin D deficiency in SLE has also been associated with higher prevalence with renal involvement $[3,26]$, photosensitive rash [26-28], leucopoenia [3], and arthritis $[27,28]$ as part of their diagnosis ACR symptom spectrum as well as flare symptoms. However within our cohort no significant association could be found with any ACR criteria and $25(\mathrm{OH}) \mathrm{D}$ levels.
ANA positivity, using a cutoff of ANA $1: 80$, was found in $80(66 \%)$ of our cohort (control (17:41\%) and SLE (63:79\%)). Unsurprisingly, a significant difference $(P=0.00)$ indicates that participants with ANA positivity are more likely to be diagnosed with autoimmune SLE. Likewise, two-sample $t$ test analysis of ANA positivity and 25(OH)D levels clearly demonstrates that lower $25(\mathrm{OH}) \mathrm{D}$ levels are associated with a greater risk of autoimmunity (Table 3 ). Our study shows that higher levels of $25(\mathrm{OH}) \mathrm{D}$ offer a significant protective effect against the expression of SLE as an illness. Of the 34 
TABLE 3: Tests of association ANA80 and $\log (2)$ transformed 25(OH)D levels.

\begin{tabular}{|c|c|c|c|c|c|c|}
\hline Test & Group $(n)$ & OR & $P>|Z|$ & Mean (SD) & & \\
\hline \multirow{3}{*}{$\begin{array}{l}\text { Two-sampled } t \text {-test } \\
\text { 25(OH)D by ANA } 80\end{array}$} & Control, SLE (121) & & 0.014 & & & \\
\hline & ANA -ve (41) & & & $68.9(21.5)$ & 62.1 & 75.7 \\
\hline & ANA +ve (80) & & & $60.3(16.9)$ & 56.5 & 64.0 \\
\hline \multirow{3}{*}{$\begin{array}{l}\text { Two-sampled } t \text {-test } \\
\log (2) 25(\mathrm{OH}) \mathrm{D} \text { by ANA } 80\end{array}$} & Control, SLE (121) & & 0.017 & & & \\
\hline & ANA -ve (41) & & & $6.0(0.45)$ & 5.9 & 6.2 \\
\hline & ANA +ve (80) & & & $5.9(0.42)$ & 5.8 & 5.9 \\
\hline \multirow{3}{*}{$\begin{array}{l}\text { Two-sampled } t \text {-test } \\
\log (2) 25(\mathrm{OH}) \mathrm{D} \text { by group }\end{array}$} & Cohort (121) & & 0.00 & & & \\
\hline & Control (41) & & & $6.1(0.42)$ & 6.0 & 6.3 \\
\hline & SLE (80) & & & $5.8(0.40)$ & 5.7 & 5.9 \\
\hline \multirow{3}{*}{$\begin{array}{l}\text { Linear regression } \\
\log (2) 25(\mathrm{OH}) \mathrm{D} \text { ANA } 80\end{array}$} & Control, SLE (121) & & 0.029 & & -0.35 & -0.02 \\
\hline & ANA -ve (41) & & & & & \\
\hline & ANA +ve (80) & & & & & \\
\hline \multirow{3}{*}{$\begin{array}{l}\text { Logistic regression } \\
\text { ANA80 } \log (2) 25(\mathrm{OH}) \mathrm{D}\end{array}$} & Control, SLE (121) & 0.36 & 0.032 & & 0.14 & 0.92 \\
\hline & ANA -ve (41) & & & & & \\
\hline & ANA +ve (80) & & & & & \\
\hline
\end{tabular}

participants showing a diagnostic deficiency of $25(\mathrm{OH}) \mathrm{D}$, $79 \%$ (27) also had ANA positivity versus ANA positivity rate of $61 \%(53)$ in the absence of $25(\mathrm{OH}) \mathrm{D}$ deficiency. Our results highlight this association further with logistic regression models indicating that for every twofold rise in vitamin $\mathrm{D}$ level, the odds ratio for autoimmunity (ANA positivity) drops to $36 \%$ of the odds ratio (OR) that existed for the baseline level.

The mean number of SRF in the SLE participants was 6.8 with 12 participants reporting no flares over the study period. SRF-day counts were calculated from the estimated number of SRF that had occurred within the preceding 12 months to interview and the estimated length of each event. Total SRF-day counts ranged from 0 to 240 days (mean 29.2 \pm 39). Two SLE participants had experienced major flares requiring extended periods in hospital, whilst 9 experienced SRF monthly and 2 on a weekly basis. The flare evaluation phase did not link individual SRF events with individual symptoms; therefore the count could represent an unresolved single symptom SRF and/or multiple events representing exacerbation of multiple or different symptoms. This study was retrospective and undertaken outside clinical assessment appointments, so validated clinic-based flare assessment tools were not able to be used.

The final pathway examined involved modelling SRF-day counts as the outcome variable and independent variables including ANA 1: 80 and ACR criteria. Due to overdispersion of SRF-day counts, a negative binomial model was undertaken. The analysis was adjusted for demographic and social factors that could exhibit confounding effects for $25(\mathrm{OH}) \mathrm{D}$ levels (age, diagnosis years, BMI, smoking, stress, vitamin D and hormone supplements, outdoor hours, and use of UV barrier products (FMSS)). Those factors significant in univariate analysis were included in the final multivariate model. Statistically significant associations for both univariate and multivariate models are listed within Table 4.
TABLE 4: Negative binomial regression for self-reported flare days (SRF-days) and independent variables.

\begin{tabular}{lcccc}
\hline & $n$ & Mean $(\mathrm{SD})$ & \multicolumn{2}{c}{$95 \%$ CI } \\
\hline SRF number (year) & 80 & $6.8(9.7)$ & 4.6 & 8.9 \\
SRF-days (year) & 80 & $29.2(39)$ & 20.5 & 37.9 \\
\hline Univariate model & OR & $P>|Z|$ & \multicolumn{2}{c}{$95 \%$ CI } \\
\hline 25(OH)D & 0.99 & 0.27 & 0.97 & 1.0 \\
Deficient 25(OH)D & 1.33 & 0.37 & 0.72 & 2.46 \\
Insufficient 25(OH)D & 0.93 & 0.82 & 0.52 & 1.67 \\
Abnormal 25(OH)D & 1.53 & 0.25 & 0.75 & 3.11 \\
Makeup (FMSS) & 1.0 & $\mathbf{0 . 0 0 2}$ & 1.0 & 1.0 \\
Diagnosis years & 0.97 & $\mathbf{0 . 0 2 4}$ & 0.94 & 1.0 \\
ANA 1: 80 & 0.42 & $\mathbf{0 . 0 0 8}$ & 0.22 & 0.80 \\
dsDNA & 0.29 & $\mathbf{0 . 0 0 9}$ & 0.12 & 0.74 \\
ENA & 0.54 & $\mathbf{0 . 0 2 7}$ & 0.31 & 0.93 \\
Arthritis & 0.48 & $\mathbf{0 . 0 2 2}$ & 0.26 & 0.90 \\
Immunological disorder & 0.49 & $\mathbf{0 . 0 0 9}$ & 0.29 & 0.84 \\
\hline Multivariate model & OR & $P>|Z|$ & \multicolumn{2}{c}{$95 \%$ CI } \\
\hline Makeup (FMSS) & 0.99 & 0.001 & 0.99 & 0.99 \\
ANA 1: 80 & 0.48 & 0.007 & 0.28 & 0.85 \\
Arthritis & 0.51 & 0.003 & 0.33 & 0.79 \\
\hline
\end{tabular}

No link was found between SRF-days and 25(OH)D levels or any sub categorisation of $25(\mathrm{OH}) \mathrm{D}$ levels indicating that whilst lower vitamin D levels may influence autoimmune diagnosis as defined by ANA-positivity, the lower levels do not influence flare risk or frequency. Protective associations were found for the use of UV barrier products of FMSS as indicated by $\mathrm{OR}$ and $P$ values in both univariate and multivariate models. However, despite a significant association between ANA positivity $(P=0.007)$, the ACR criterion of arthritis $(P=0.003)$, and SRF frequency, this association was in a negative direction with $\mathrm{OR}$ of 0.48 and 0.51 , respectively. 


\section{Discussion}

4.1. Vitamin D Levels of Deficiency. Deficiency in vitamin $\mathrm{D}$ levels is often reported in general populations and is increasingly linked to increased incidence of autoimmune illnesses such as SLE and other musculoskeletal illnesses [12, 29]. General annual Australian population rates of deficiency are reported at $6 \%$ [30], with an estimate of $23 \%$ having levels below recommended "normal" levels (insufficient + deficient). Rates of deficiency vary significantly across seasons with rates in summer being lower than those in winter. Summer rates in the state of NSW are $19 \%$ with little variation with gender [30]. The proportion of $25(\mathrm{OH}) \mathrm{D}$ deficient but otherwise healthy individuals within our cohort was $14.6 \%$ as compared to our SLE group with a deficiency in $35.0 \%$. Significant differences between our cohort groups were found and are reflective of similar findings internationally [26, 27]; however the proportion of deficiency found within our cohort differed from other studies with deficiency proportions within the SLE patients of $8.2 \%$ [31] to $20 \%[3,14]$ although one study reported prevalence as high as $67 \%$ [32]. Variations in deficiency prevalence may be derived from population differences including geographic location, diet, social and economic factors, and population ethnicity. Significantly higher deficiency levels are often reported within individuals of African, Asian, and African American background and, in particular, persons with darker skin complexions $[28,33,34]$.

Levels of insufficiency within our cohort were found in both healthy (46.3\%) and SLE (48.8\%) groups without adjustment for supplementation. This finding was considerably reduced when adjusted for supplement use. Insufficiency is not an unusual finding in patients with SLE even with supplementation [31, 35] with suggestions of a worldwide vitamin D insufficiency epidemic with levels falling $20 \%$ in 2002-2012 [36]. Our results suggest that within the Australian population there is also a high proportion of women with inadequate levels of $25(\mathrm{OH}) \mathrm{D}$ at either a deficient or insufficient level, with this inadequacy heightened within the autoimmune SLE population. Further to this the benefits of supplementation, which did not significantly differ across the groups, appear to be reduced within the autoimmune SLE population, supporting the need for additional or nonstandard measures of support and monitoring. Beneficial supplementation to raise $25(\mathrm{OH}) \mathrm{D}$ levels to above insufficient levels within an SLE patient has been reported within a phase 1 trial to require daily doses of 2000 IU as opposed to current standard doses of 600-800 IU [37].

4.2. Expression of Autoimmunity (ANA 1:80 Positivity). It is reported that $13-45 \%$ of healthy individuals may have ANA positivity during their lifetime [38-40]. Prevalence is reported to be higher with aging and in women. High variation in frequency ranges has also been found across various geographical groups and with methods [41]. Within our cohort, ANA positivity with a dilution cutoff at $1: 80$ was found in $41 \%$ of our controls (without autoimmune illness) as compared to $79 \%$ in ACR confirmed SLE patients with a significance difference in prevalence found between the two groups. Prevalence within healthy controls was at the upper end of our expectation and reflected the high proportion of older individuals $(9 / 17=53 \%$ over 50 years $)$ in the study. A high ANA positivity prevalence had previously been found in healthy controls in a Hunter, NSW community cohort study Boyle [42].

4.3. Vitamin D and Expression of Autoimmunity (ANA 1:80 Positivity). The log2 logistic model related 25(OH)D levels to probability of ANA positivity. Substitution allowed absolute odds values to be calculated. For a 25(OH)D level of $40 \mathrm{nmol} / \mathrm{L}$, the probability of ANA positivity is $79 \%$ (odds $3.67: 1)$, whilst a $25(\mathrm{OH}) \mathrm{D}$ level of $80 \mathrm{nmol} / \mathrm{L}$ is associated with an ANA positivity probability of only $57 \%$ (odds $1.32: 1$ ), equating to $36 \%$ of the odds of ANA positivity for the lower vitamin $\mathrm{D}$ level of 40 (equivalent to a relative risk reduction of $67 \%$ ). This confirms that as vitamin D levels increase, probability of autoimmune disease expression decreases. This finding, with particular reference to vitamin D deficiency and expression of an autoimmune disease (ANA 1:80), is in line with established literature $[43,44]$.

4.4. Clinical Autoimmunity (ANA 1:80 Positivity) and SelfReported Flare. The negative association between serological magnitude of autoimmunity and SRF frequency was unexpected but consistently demonstrated throughout data subanalysis of ANA patterns (homogeneous only), titres, and a restricted analysis of ANA-positive participants with concurrent ENA and/or DNA positivity. ANA patterns were of particular interest due to reports of dense fine speckled 70 (DFS70) antigen staining, as a potential marker of protection against autoimmunity $[39,45,46]$.

This concords with evidence for SLE and autoimmunity representing a multistep process proceeding from selftolerance to serological "benign" autoimmunity (step 1), followed by later conversion of serological "silent" autoimmunity, to overt autoimmune disease (SLE) (step 2) [47]. Current evidence [40] supports the contention that the triggers invoking each of these transitions (steps 1 and 2) are distinct for each step, with basic immunomodulating factors such as vitamin $\mathrm{D}$ levels being associated with transition across the first step in autoimmune pathogenesis and with other factors, perhaps impacting upon maintenance of peripheral immune regulation (e.g., hormones, sunlight) being important in driving step 2 .

Given the virtually universal requirement for ANA positivity to exist before a clinical diagnosis of SLE is made, there is an inherent incorporation bias operating in any study attempting to tease out relative pathogenic contributions of various determinants to either "benign" (serological) or "overt" (clinical) autoimmunity: this is because the presence of ANA positivity increases the likelihood of a SLE diagnosis being made. This phenomenon occurs despite the decline in immune function with aging (immunosenescence), which is often associated with a rise in ANA expression and titres, in the absence of any clinical autoimmune disease presence.

Given the results of separate studies defining links between environmental chemical exposure and SRF frequency $[48,49]$, the proposal that different triggers operate 
for each transitional step seems appropriate and consistent with available data.

The phenomenon of "protective" autoimmunity is well established, with antinuclear antibodies having been shown to operate to limit immune-related pathology in a range of settings. Examples of protective autoimmunity include (i) expression of ENA along with ANA protecting against severe renal lupus [50]; (ii) antibodies to high-mobility group protein B1 (HMGB1) ANA, attenuating disease in mouse models and providing survival benefit in patients with septic shock [40], suggesting that infection-induced antibodies may be beneficial in some instances; and (iii) "DFS- (dense fine speckled-) 70" antibodies (previously labelled as a type of homogeneous ANA with speckling) which are increasingly reported in clinical studies, and there is a reported reduction in prevalence of lupus-related autoimmune syndromes in this patient subgroup [38, 39].

Our study's findings are consistent with a proposed role of ANA in offering protection against lupus flares, and, indeed, expression of some ANAs may be a marker of adaptive regulatory immune function as an allostatic mechanism.

4.5. Vitamin D and Self-Reported Flare. Despite the clear autoimmune predisposition conferred by low $25(\mathrm{OH}) \mathrm{D}$ levels, we could find no significant association between $25(\mathrm{OH}) \mathrm{D}$ levels and frequency of SRF in SLE participants. A lack of correlation has also been reported in other vitamin D/activity studies $[31,51]$. In contrast, a large number of studies have found inverse relationships between low levels of $25(\mathrm{OH}) \mathrm{D}$ and SLE activity measures [23, 26, 34, 45]. Within our study we propose that a number of factors may explain our lack of association, including (i) lack of statistical study power; (ii) retrospective nature of SRF; and (iii) reasoning that vitamin $\mathrm{D}$ deficiency may predispose to disease expression without influencing its severity or behaviour once established. As discussed above, the two-step model of conversion from tolerance to "benign" autoimmunity and then to "clinical" autoimmunity is consistent with separate roles for different determining factors in the expression of aberration. Thus, whilst we have shown that vitamin D levels have impact upon step 1 (the expression of "benign" autoimmunity), we must posit other factors that prompt the second step of autoimmune disease development, possibly triggered by other environmental or hormonal factors $[48,49$, 52].

\section{Study Strengths and Weaknesses}

This study provides retrospective data within the context of a relatively homogenous Caucasian population with a validated SLE diagnosis according to ACR criteria. The retrospective design would limit firm establishment of casual relationships. The study was undertaken outside routine clinical management appointments with data collection from a nonclinician; therefore SLE flare data collected was from a self-reported patient perspective rather than according to standard disease activity measures. To minimise bias, the methods followed a standardised protocol which included a flare definition [21] and structured interview method [18]. Individual SRF events were not matched to specified symptoms and without clarification of whether full resolution of flare occurred each time an event was reported; however only a few participants reported flare frequency rates greater than monthly. The selfreported nature of flare assessment may have resulted in an overestimation of actual days and events recorded creating potential bias against detection of a significant association with vitamin $\mathrm{D}$ levels.

Despite collection of samples within a single spring season, the dynamic and fluctuating nature of vitamin D levels [34] coupled with the retrospective assessment of SRF may have resulted in missed associations between $25(\mathrm{OH}) \mathrm{D}$ and flare frequency.

The capacity for extrapolation of results from this study is limited by the lack of diversity in ethnicity and gender within our study population. The results should be viewed as of a pilot nature with the need for a comprehensive prospective study protocol documenting vitamin $\mathrm{D}$ levels over time along with assessment of disease activity and occurrence of flares potentially adding to evidence of any associations.

\section{Conclusion}

In summary, our paper reinforces existing literature supporting a link between vitamin D deficiency and the expression of autoimmune phenomena (ANA positivity) and clinical conditions (such as SLE). In contrast, there was no demonstrable association between vitamin D levels and flare frequency, consistent with the proposed independent influence of other flare-inducing factors. The results indicate that SLE patients are at a high risk of vitamin $\mathrm{D}$ deficiency and vitamin $\mathrm{D}$ supplementation along with regular monitoring should be a consideration as part of individual patient health management plans.

\section{Conflict of Interests}

The authors declare that there is no conflict of interests regarding the publication of this paper.

\section{Acknowledgments}

This study forms a part of the Environmental Determinants of Lupus Flare (EDOLF) Ph.D. study, University of Newcastle. The study was funded via resources provided by the Autoimmune Resource Research Centre (not-forprofit charity http://www.autoimmune.org.au) and the Val Badham Research Scholarship for Immunology, University of Newcastle Foundation. The study team would also like to acknowledge the contribution from Pathology Service staff (Pathology North Hunter New England) of the Clinical Chemistry and Immunology Departments, in particular, Robert Lojewski.

\section{References}

[1] C. C. Mok and C. S. Lau, "Pathogenesis of systemic lupus erythematosus," Journal of Clinical Pathology, vol. 56, no. 7, pp. 481-490, 2003. 
[2] G. S. Cooper, J. Wither, S. Bernatsky et al., "Occupational and environmental exposures and risk of systemic lupus erythematosus: silica, sunlight, solvents," Rheumatology, vol. 49, no. 11, Article ID keq214, pp. 2172-2180, 2010.

[3] J. Bogaczewicz, A. Sysa-Jedrzejowska, C. Arkuszewska et al., "Vitamin D status in systemic lupus erythematosus patients and its association with selected clinical and laboratory parameters," Lupus, vol. 21, no. 5, pp. 477-484, 2012.

[4] L. M. Vilá, A. M. Mayor, A. H. Valentín et al., "Association of sunlight exposure and photoprotection measures with clinical outcome in systemic lupus erythematosus," Puerto Rico Health Sciences Journal, vol. 18, no. 2, pp. 89-94, 1999.

[5] J. F. Carvalho, M. Blank, E. Kiss, T. Tarr, H. Amital, and Y. Shoenfeld, "Anti-vitamin D, vitamin D in SLE: preliminary results," Annals of the New York Academy of Sciences, vol. 1109, pp. 550-557, 2007.

[6] M. C. Borges, L. A. Martini, and M. M. Rogero, "Current perspectives on vitamin D, immune system, and chronic diseases," Nutrition, vol. 27, no. 4, pp. 399-404, 2011.

[7] F. Baeke, T. Takiishi, H. Korf, C. Gysemans, and C. Mathieu, "Vitamin D: modulator of the immune system," Current Opinion in Pharmacology, vol. 10, no. 4, pp. 482-496, 2010.

[8] X. Guillot, L. Semerano, N. Saidenberg-Kermanac'h, G. Falgarone, and M.-C. Boissier, "Vitamin D and inflammation," Joint Bone Spine, vol. 77, no. 6, pp. 552-557, 2010.

[9] M. Wacker and M. F. Holick, "Sunlight and Vitamin D: a global perspective for health," Dermato-Endocrinology, vol. 5, no. 1, pp. 51-108, 2013.

[10] M. Racovan, B. Walitt, C. E. Collins et al., "Calcium and vitamin $\mathrm{D}$ supplementation and incident rheumatoid arthritis: the Women's Health Initiative Calcium plus Vitamin D trial," Rheumatology International, vol. 32, no. 12, pp. 3823-3830, 2012.

[11] M. Cutolo, C. Pizzorni, and A. Sulli, "Vitamin D endocrine system involvement in autoimmune rheumatic diseases," Autoimmunity Reviews, vol. 11, no. 2, pp. 84-87, 2011.

[12] A. Kumar, H. Gopal, K. Khamkar et al., "Vitamin D deficiency as the primary cause of musculoskeletal complaints in patients referred to rheumatology clinic: a clinical study," Indian Journal of Rheumatology, vol. 7, no. 4, pp. 199-203, 2012.

[13] H. L. Miller, F. A. Farraye, J. Coukos et al., "Insufficiency are common in ulcerative colitis patients after ileal pouch-anal anastomosis," Inflammatory Bowel Diseases, vol. 19, no. 2, pp. E25-E26, 2013.

[14] C. F. Pelajo, J. M. Lopez-Benitez, and L. C. Miller, "Vitamin $\mathrm{D}$ and autoimmune rheumatologic disorders," Autoimmunity Reviews, vol. 9, no. 7, pp. 507-510, 2010.

[15] P. Pludowski, M. F. Holick, S. Pilz et al., "Vitamin D effects on musculoskeletal health, immunity, autoimmunity, cardiovascular disease, cancer, fertility, pregnancy, dementia and mortality-a review of recent evidence," Autoimmunity Reviews, vol. 12, no. 10, pp. 976-989, 2013.

[16] Rheumatology ACo, "Classification criteria for diagnosis of systemic lupus erythematosus," 1997 Update of the 1982 American College of Rheumatology Revised Criteria for Classification of Systemic Lupus Erythematosus, 2005, http://www.rheumatology.org/practice/clinical/classification/ sle/1997_update_of_the_1982_American_College_of_Rheumatology_Revised_Criteria_for_Classification_of_sle.pdf.

[17] V. Parravicini and S. Caserta, "The immunomodulatory roles of vitamin D: new tricks for an old dog," Molecular Interventions, vol. 10, no. 4, pp. 204-208, 2010.
[18] M. L. Squance, M. Guest, G. Reeves, J. Attia, and H. Bridgman, "Exploring lifetime occupational exposure and SLE flare: a patient-focussed pilot study," Lupus Science \& Medicine, vol. 1, no. 1, Article ID e000023, 2014.

[19] Association WM, World Medical Association Declaration of Helsinki, Declaration of Helsinki 2008 revision, 2008, http://www.wma.net/en/30publications/10policies/b3/17c.pdf.

[20] NSW Department of Health, New South Wales Population Health Survey 2010, Centre for Epidemiology and Research PHD, NSW Department of Health, Eds., NSW Government, Sydney, Australia, 2011.

[21] C. M. Poser, D. W. Paty, and L. Scheinberg, "New diagnostic criteria for multiple sclerosis: guidelines for research protocols," Annals of Neurology, vol. 13, no. 3, pp. 227-231, 1983.

[22] Household Products Database, 2013, http://householdproducts. nlm.nih.gov/index.htm.

[23] A. Doria, S. Arienti, M. Rampudda, M. Canova, M. Tonon, and P. Sarzi-Puttini, "Preventive strategies in systemic lupus erythematosus," Autoimmunity Reviews, vol. 7, no. 3, pp. 192$197,2008$.

[24] Meteorology AGBo, Climate data for Hunter and Central Coast Region (Sept-Nov 2006), Canberra, Australia, Commonwealth of Australia, 2014, http://www.bom.gov.au/ climate/cdo/about/austmaps/solar-radiation-glossary.shtml.

[25] Australia CC, Health promotion: sun protection advice, 2014, http://www.cancer.org.au/preventing-cancer/sun-protection/ sun-protection-products/.

[26] D. L. Kamen, G. S. Cooper, H. Bouali, S. R. Shaftman, B. W. Hollis, and G. S. Gilkeson, "Vitamin D deficiency in systemic lupus erythematosus," Autoimmunity Reviews, vol. 5, no. 2, pp. 114-117, 2006.

[27] M. Barbhaiya and K. H. Costenbader, "Ultraviolet radiation and systemic lupus erythematosus," Lupus, vol. 23, no. 6, pp. 588595, 2014.

[28] A. Duarte-García, H. Fang, C. H. To, L. S. Magder, and M. Petri, "Seasonal variation in the activity of systemic lupus erythematosus," Journal of Rheumatology, vol. 39, no. 7, pp. 1392-1398, 2012.

[29] A. Hajas, J. Sandor, L. Csathy et al., "Vitamin D insufficiency in a large MCTD population," Autoimmunity Reviews, vol. 10, no. 6, pp. 317-324, 2011.

[30] Statistics ABo, Australian Health Survey: Biomedical Results for Nutrients, 2011-2012, ABS, Canberra, Australia, 2012.

[31] M. I. D. Souto, A. Coelho, C. Guo et al., "Vitamin D insufficiency in Brazilian patients with SLE: prevalence, associated factors, and relationship with activity," Lupus, vol. 20, no. 10, pp. 10191026, 2011.

[32] J. D. Alele and D. L. Kamen, "The importance of inflammation and vitamin D status in SLE-associated osteoporosis," Autoimmunity Reviews, vol. 9, no. 3, pp. 137-139, 2010.

[33] M. Hewison, "Vitamin D and the immune system: new perspectives on an old theme," Rheumatic Disease Clinics of North America, vol. 38, no. 1, pp. 125-139, 2012.

[34] D. J. Birmingham, L. A. Hebert, H. Song et al., "Evidence that abnormally large seasonal declines in vitamin D status may trigger SLE flare in non-African Americans," Lupus, vol. 21, no. 8, pp. 855-864, 2012.

[35] S. M. A. Toloza, D. E. C. Cole, D. D. Gladman, D. Ibañez, and M. B. Urowitz, "Vitamin D insufficiency in a large female SLE cohort," Lupus, vol. 19, no. 1, pp. 13-19, 2010. 
[36] M. F. Holick, "Vitamin D deficiency," The New England Journal of Medicine, vol. 357, no. 3, pp. 266-281, 2007.

[37] A. C. Ross, J. E. Manson, S. A. Abrams et al., “The 2011 report on dietary reference intakes for calcium and vitamin $\mathrm{D}$ from the Institute of Medicine: what clinicians need to know," Journal of Clinical Endocrinology and Metabolism, vol. 96, no. 1, pp. 53-58, 2011.

[38] C. Fitch-Rogalsky, W. Steber, M. Mahler et al., "Clinical and serological features of patients referred through a rheumatology triage system because of positive antinuclear antibodies," PLoS ONE, vol. 9, no. 4, Article ID e93812, 2014.

[39] M. Mahler, J. G. Hanly, and M. J. Fritzler, "Importance of the dense fine speckled pattern on HEp-2 cells and anti-DFS70 antibodies for the diagnosis of systemic autoimmune diseases," Autoimmunity Reviews, vol. 11, no. 9, pp. 642-645, 2012.

[40] D. S. Pisetsky, "Antinuclear antibodies in rheumatic disease: a proposal for a function-based classification," Scandinavian Journal of Immunology, vol. 76, no. 3, pp. 223-228, 2012.

[41] Y. Shapira, B.-S. P. Katz, B. Gilburd et al., "Geographical differences in autoantibodies and anti-infectious agents antibodies among healthy adults," Clinical Reviews in Allergy \& Immunology, vol. 42, no. 2, pp. 154-163, 2012.

[42] M. Boyle, Hunter NSW Community Cohort study-ANA Positivity, Personal Communication with G. Reeves, Newcastle, UK, unpublished study of study, 2014.

[43] M. A. Kriegel, J. E. Manson, and K. H. Costenbader, "Does vitamin $\mathrm{D}$ affect risk of developing autoimmune disease?: a systematic review," Seminars in Arthritis and Rheumatism, vol. 40, no. 6, pp. 512.e8-531.e8, 2011.

[44] L. L. Ritterhouse, S. R. Crowe, T. B. Niewold et al., "Vitamin D deficiency is associated with an increased autoimmune response in healthy individuals and in patients with systemic lupus erythematosus," Annals of the Rheumatic Diseases, vol. 70, no. 9, pp. 1569-1574, 2011.

[45] H. Amital, Z. Szekanecz, G. Szücs et al., "Serum concentrations of $25-\mathrm{OH}$ vitamin $\mathrm{D}$ in patients with systemic lupus erythematosus (SLE) are inversely related to disease activity: is it time to routinely supplement patients with SLE with vitamin D?" Annals of the Rheumatic Diseases, vol. 69, no. 6, pp. 1155-1157, 2010.

[46] A. Doria, M. Zen, M. Canova et al., "SLE diagnosis and treatment: when early is early," Autoimmunity Reviews, vol. 10, no. 1, pp. 55-60, 2010.

[47] G. E. M. Reeves, "Update on the immunology, diagnosis and management of systemic lupus erythematosus," Internal Medicine Journal, vol. 34, no. 6, pp. 338-347, 2004.

[48] E. V. Hess, "Environmental chemicals and autoimmune disease: cause and effect," Toxicology, vol. 181-182, pp. 65-70, 2002.

[49] M. L. Squance, Everyday products and SLE impacts on patient perceived flare frequency [Manuscript Research Ph.D. thesis], 2014.

[50] F. J. Tápanes, M. Vásquez, R. Ramírez, C. Matheus, M. A. Rodríguez, and N. Bianco, "Cluster analysis of antinuclear autoantibodies in the prognosis of SLE nephropathy: are antiextractable nuclear antibodies protective?" Lupus, vol. 9, no. 6, pp. 437-444, 2000.

[51] K. Müller, N. J. Kriegbaum, B. Baslund, O. H. Sørensen, M. Thymann, and K. Bentzen, "Vitamin D3 metabolism in patients with rheumatic diseases: Low serum levels of 25hydroxyvitamin D3 in patients with systemic lupus erythematosus," Clinical Rheumatology, vol. 14, no. 4, pp. 397-400, 1995.
[52] F. W. Miller, "Environmental agents and autoimmune diseases," Advances in Experimental Medicine and Biology, vol. 711, pp. 6181, 2011. 


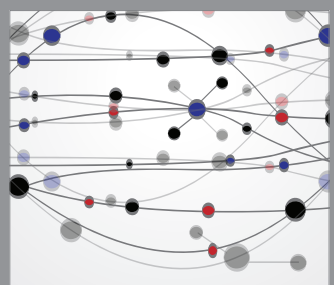

The Scientific World Journal
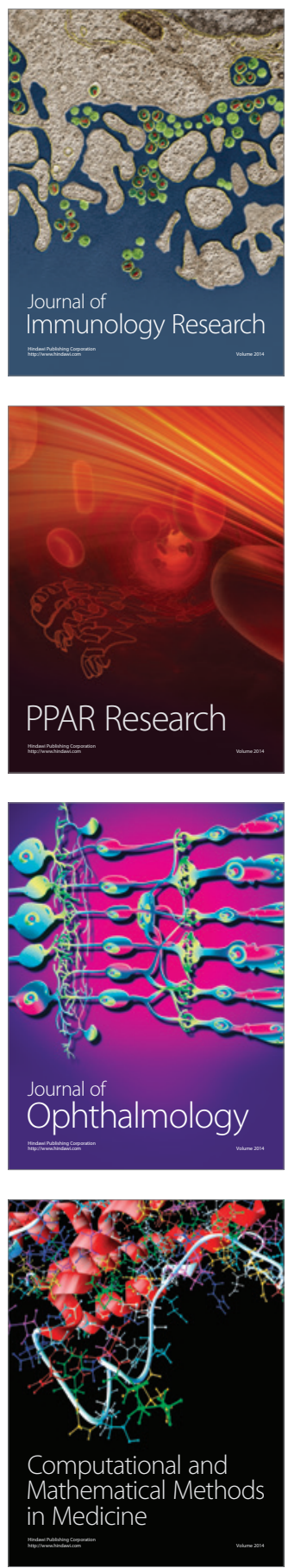

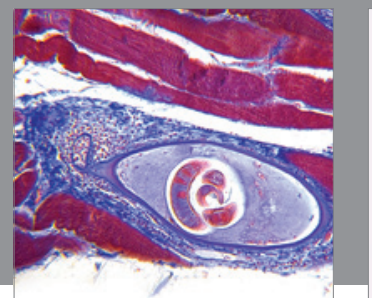

Gastroenterology

Research and Practice
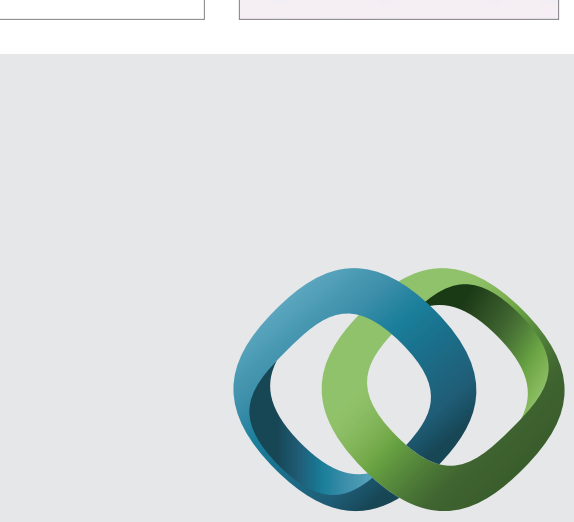

\section{Hindawi}

Submit your manuscripts at

http://www.hindawi.com
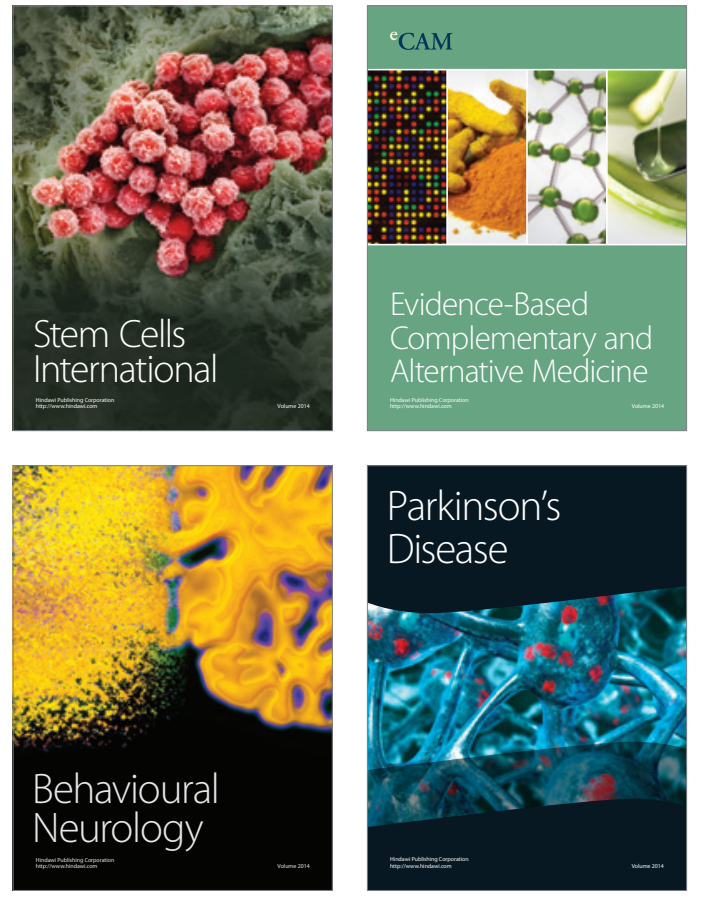
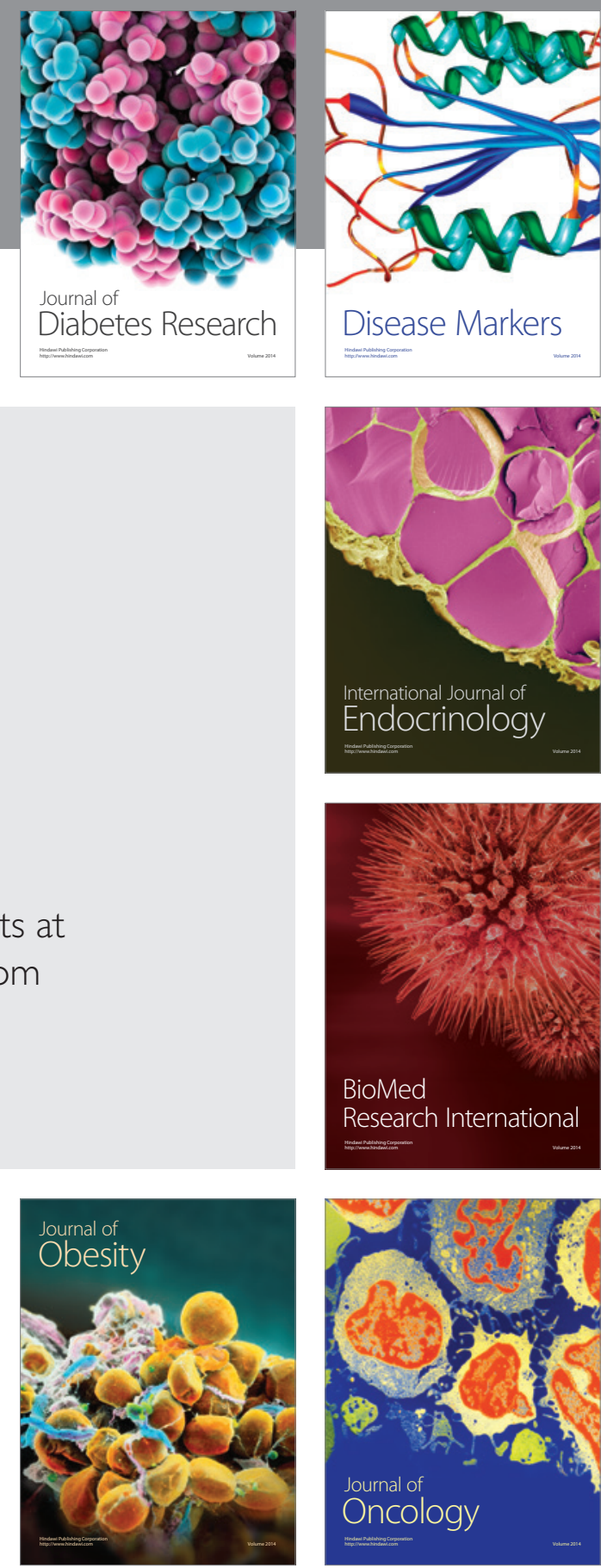

Disease Markers
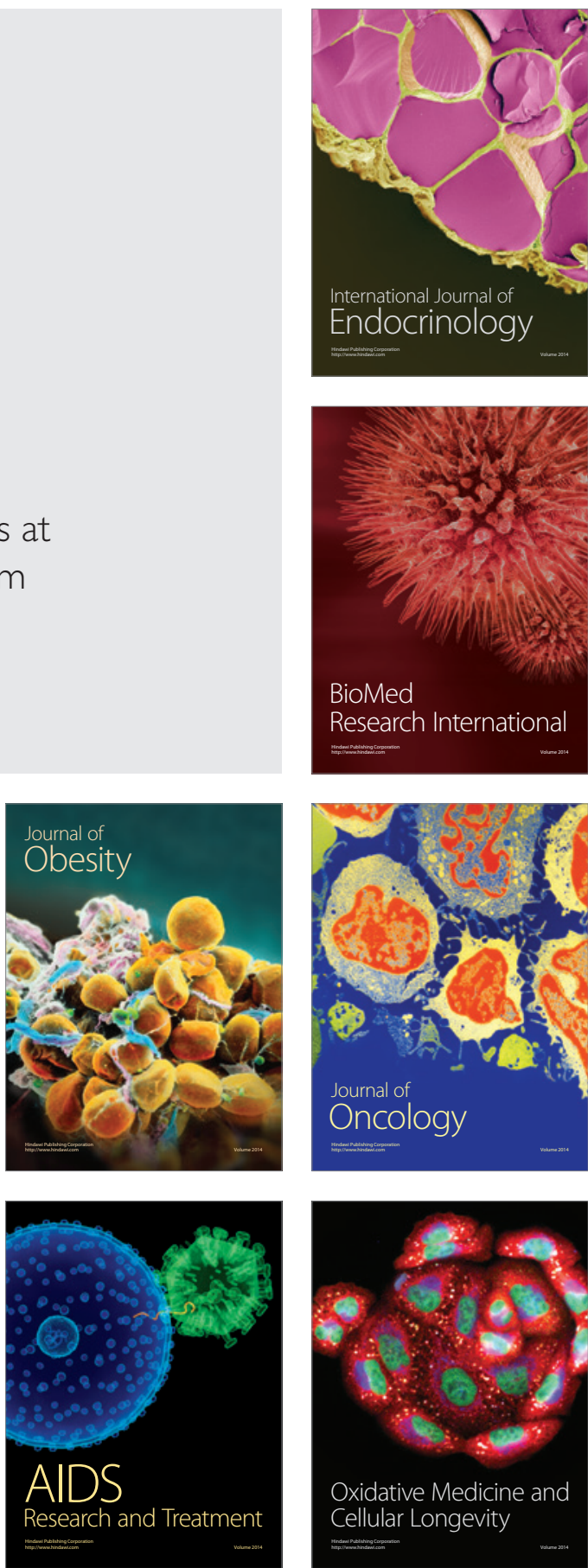